\title{
Free vibration of frame structures made of Zener type viscoelastic material
}

\author{
Roman Lewandowski ${ }^{1, *}$, and Przemystaw Wielentejczyk ${ }^{2}$ \\ ${ }^{1}$ Poznan University of Technology, Institute of Structural Engineering, 60-965 Poznan, Poland \\ ${ }^{2}$ NEOKON, Construction Design Office, ul. Miodowa 12, 60-591 Poznan, Poland
}

\begin{abstract}
A method for determining the dynamic characteristics of structures made of viscoelastic material is presented. The fractional Zener model is used to the describe the rheological properties of materials. All of the elements of a structure must be built of material with identical rheological properties. The solution to the linear eigenvalue problem for some elastic structure and the solution to a single nonlinear algebraic equation are needed to obtain the dynamic characteristics of a viscoelastic structure. Moreover, the frequency response functions are determined in a very efficient way. The results of a representative calculation are presented and briefly discussed.
\end{abstract}

\section{Introduction}

The natural frequencies, non-dimensional damping ratios, modes of vibration and frequency response functions are very important characteristics of each mechanical structure. For elastic systems, these properties can be determined using well-known methods, described in many monographs (see, for example [1, 2]). However, methods which are devoted to determining the dynamic properties of viscoelastic (VE) structures or elastic structures with viscoelastic dampers also exist (see, for example [3 - 5]) but they do not fully clarify a number of problems. For this type of structures, the dynamic characteristics are obtained by solving a nonlinear eigenvalue problem. In some cases, when the VE materials or dampers are described using the classic rheological models, the above-mentioned nonlinear eigenvalue problem can be transformed into the linear one after introducing the concept of state and/or internal variables $[2,6]$. The methods used to solve the nonlinear eigenvalue problem include the continuation method [3, 4], Newton's eigenvalue iteration method [7], the iterative method [5] and some other ones.

In this paper, the dynamic characteristics of VE frame structures made of a material described by the fractional Zener model are analysed. It is assumed that the whole structure is homogeneous and made of a material described by a fractional Zener model and the parameters of such model are identical for all the elements of the structure. In particular, the new method for solving the nonlinear eigenvalue problem, of which the computational efficiency is very high, is presented. In the method, it is necessary to know a solution to the linear eigenvalue problem for some elastic structures and a solution to a single algebraic equation to determine the dynamic characteristics of the VE structures. However, the

* Corresponding author: roman.lewandowski@put.poznan.pl 
presented method can be used only to analyse the above-mentioned homogeneous VE structures. The frequency response functions (FRF) are determined in a very efficient way.

\section{Equation of motion for a rod treated as a continuous system}

The Euler theory is used to describe the dynamic behaviour of rods made of viscoelastic (VE) materials. The well-known equilibrium equations are:

$$
\begin{gathered}
-N_{, x}(x, t)+m \ddot{u}(x, t)=p_{x}(x, t), \\
-T_{, x}(x, t)+m \ddot{w}(x, t)=p_{y}(x, t), \quad T(x, t)=M_{, x}(x, t),
\end{gathered}
$$

where $M(x, t), T(x, t)$ and $N(x, t)$ are the bending moments, the shear and normal forces, respectively; $u(x, t), w(x, t)$ are the axial and transversal displacements of the neutral axis of the rods; $p_{x}(x, t)$ and $p_{y}(x, t)$ are the axial and transversal excitation forces per unit length of a rod and $m$ is the mass per unit length of the rod. The dot indicates differentiation with respect to time $t$ and $(\bullet)_{, x}(x, t)=d(\bullet) / d x$.

The axial strain of the neutral axis of the $\operatorname{rod} \varepsilon(x, t)$, the angle of rotation of the rod's cross-section $\varphi(x, t)$ and the rod's curvature $\kappa(x, t)$ are defined as:

$$
\varphi(x, t)=w_{, x}(x, t), \quad \kappa(x, t)=-w_{, x x}(x, t), \quad \varepsilon(x, t)=u_{, x}(x, t) .
$$

The constitutive relationships for the viscoelastic material of Zener type written at the rod's cross-section level take the following form [8]:

$$
\begin{aligned}
& M(x, t)+\tau^{\alpha} \mathrm{D}_{t}^{\alpha} M(x, t)=E_{0} J \kappa_{x}(x, t)+\tau^{\alpha} E_{\infty} J \mathrm{D}_{t}^{\alpha} \varepsilon_{x}(x, t), \\
& N(x, t)+\tau^{\alpha} \mathrm{D}_{t}^{\alpha} N(x, t)=E_{0} A \varepsilon(x, t)+\tau^{\alpha} E_{\infty} A \mathrm{D}_{t}^{\alpha} \varepsilon(x, t),
\end{aligned}
$$

where $A$ and $J$ are the rod's cross-sectional area and the moment of inertia of the crosssection. Moreover, $E_{0}, E_{\infty}$ and $\tau$ are the relaxed and non-relaxed elastic modulus and the relaxation time, respectively. $\mathrm{D}_{t}^{\alpha} x(t)$ is the Riemann-Liouville fractional derivative of order $\alpha$ of function $x(t)$ with respect to time.

The governing relationships of the non-viscously damped systems are often defined in the frequency domain. For this reason, Eqns (4) and (5) can be equivalently expressed in the Laplace domain (assuming zero initial conditions) as follows:

$$
\bar{N}(x, s)=s \bar{G}(s) A \bar{\varepsilon}(x, s), \quad \bar{M}(x, s)=s \bar{G}(s) J \bar{\kappa}(x, s),
$$

where $s$ is the Laplace variable and $s \bar{G}(s)$ is the Laplace transform of the heredity function. The particular form of the $s \bar{G}(s)$ function for the Zener model of material is:

$$
s \bar{G}(s)=\left(1+\frac{\tau^{\alpha}{ }^{\alpha}{ }^{\alpha}}{1+\tau^{\alpha}{ }^{\alpha}} \frac{\Delta E}{E_{0}}\right) E_{0}=(1+\theta(s)) E_{0},
$$

where $\Delta E=E_{\infty}-E_{0}$.

The Laplace transformation of Eqs (1) - (3) give us: 


$$
\begin{aligned}
&-\bar{N}_{, x}(x)+s^{2} m \bar{u}(x)= \bar{p}_{x}(x), \quad-\bar{T}_{, x}(x)+s^{2} m \bar{w}(x)=\bar{p}_{y}(x), \\
& \bar{T}(x)=\bar{M}_{, x}(x), \\
& \bar{\varphi}(x)=\bar{w}_{, x}(x), \quad \bar{\kappa}(x)=-\bar{w}_{, x x}(x), \quad \bar{\varepsilon}(x)=\bar{u}_{, x}(x),
\end{aligned}
$$

where the quantities with the bar are the Laplace transforms of the corresponding quantities without the bar.

\section{The finite element formulation for a viscoelastic rod}

The finite element method is a general and widely-accepted method to solve many dynamic problems. Here, the planar, two-node rod element with six nodal parameters and the Hermite shape functions, shown in Fig. 1, are used to describe the dynamic behaviour of a viscoelastic rod.

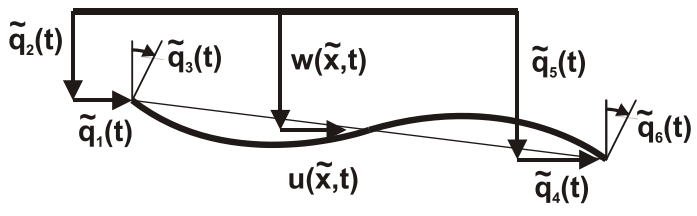

Fig. 1. Schematic of the planar finite element.

The vector of nodal parameters (in a local coordinate axis) is $\mathbf{q}_{e}(t)=\left[\widetilde{q}_{1}(t), \widetilde{q}_{2}(t), \widetilde{q}_{3}(t), \widetilde{q}_{4}(t), \widetilde{q}_{5}(t), \widetilde{q}_{6}(t)\right]^{T}$ (see Fig.1). The vector of displacements defined in the time domain is $w_{e}(x, t)=[u(x, t), w(x, t)]^{T}$ and defined in the Laplace domain $\overline{\mathbf{w}}_{e}(x, s)=[\bar{u}(x, s), \bar{w}(x, s)]^{T}$ is written in terms of nodal parameters in the following way:

$$
\mathbf{w}_{e}(x, t)=\mathbf{H}(x) \mathbf{q}_{e}(t), \quad \overline{\mathbf{w}}_{e}(x, s)=\mathbf{H}(x) \overline{\mathbf{q}}_{e}(s),
$$

where $\mathbf{H}(x)$ is the well-known matrix of shape functions [2]. The vector of generalized strains $\mathbf{e}(x, t)=[\varepsilon(x, t), \kappa(x, t)]^{T}$ and his Laplace transform $\overline{\mathbf{e}}(x, s)=[\bar{\varepsilon}(x, s), \bar{\kappa}(x, s)]^{T}$ depends on the nodal parameters as follows:

$$
\mathbf{e}(x, t)=\mathbf{B}(x) \mathbf{q}_{e}(t), \quad \overline{\mathbf{e}}(x, s)=\mathbf{B}(x) \overline{\mathbf{q}}_{e}(s),
$$

where $\mathbf{B}(x)$ is the matrix containing appropriate derivatives of the shape functions [2].

In both of the above-mentioned domains, the vector of excitation forces is defined as $\mathbf{p}_{e}(x, t)=\left[p_{x}(x, t), p_{y}(x, t)\right]^{T}$ and $\overline{\mathbf{p}}_{e}(x, s)=\left[\bar{p}_{x}(x, s), \bar{p}_{y}(x, s)\right]^{T}$. Moreover, the vectors of inertia forces $\mathbf{b}_{e}(x, t)=\left[b_{x}(x, t), b_{y}(x, t)\right]^{T}$ and $\overline{\mathbf{b}}_{e}(x, s)=\left[\bar{b}_{x}(x, s), \bar{b}_{y}(x, s)\right]^{T}$ depend on nodal acceleration in the usual way

$$
\mathbf{b}_{e}(x, t)=-m \mathbf{H}(x) \ddot{\mathbf{q}}_{e}(t), \quad \overline{\mathbf{b}}_{e}(x, s)=-s^{2} m \mathbf{H}(x) \overline{\mathbf{q}}_{e}(s) .
$$

After introducing the vector of generalized stresses $\mathbf{s}(x, t)=[N(x, t), M(x, t)]^{T}$, the matrix form of the constitutive relationships (6) is:

$$
\overline{\mathbf{s}}(x, s)=s \overline{\mathbf{G}}(s) \overline{\mathbf{e}}(x, s),
$$


where

$$
s \overline{\mathbf{G}}(s)=s \bar{G}(s)\left[\begin{array}{cc}
A & 0 \\
0 & J
\end{array}\right] .
$$

Using the above notations, the virtual work equation for the finite element could be written as follows:

$$
\delta \mathbf{q}_{e}^{T}(t) \mathbf{R}_{e}(t)+\int_{0}^{l_{e}} \delta \mathbf{w}_{e}^{T}(x, t)\left(\mathbf{p}_{e}(x, t)+\mathbf{b}_{e}(x, t)\right) d x=\int_{0}^{l_{e}} \delta \mathbf{e}^{T}(x, t) \mathbf{s}(x, t) d x d t,
$$

where the symbols $\delta \mathbf{w}_{e}(x, t), \delta \mathbf{q}_{e}(t)$ and $\delta \mathbf{e}(x, t)$ denote the vector of virtual displacements, the vector of virtual nodal displacements and the vector of virtual generalized strains, respectively. Moreover, $\mathbf{R}_{e}(t)=\left[R_{1}(t), R_{2}(t), R_{3}(t), R_{4}(t), R_{5}(t), R_{6}(t)\right]^{T}$ is the vector of nodal reactions and $l_{e}$ is the length of the finite element.

In the Laplace's domain, the virtual work equation (16) takes the following form:

$$
\delta \overline{\mathbf{q}}_{e}^{T} \overline{\mathbf{R}}_{e}+\int_{0}^{l_{e}} \delta \overline{\mathbf{w}}_{e}^{T}(x)\left(\overline{\mathbf{p}}_{e}(x)+\overline{\mathbf{b}}_{e}(x)\right) d x=\int_{0}^{l_{e}} \delta \overline{\mathbf{e}}^{T}(x) \overline{\mathbf{s}}(x) d x .
$$

Proceeding in the usual way, the second and third terms of Eq. (17) can be rearranged in the following way:

$$
\int_{0}^{l_{e}} \delta \overline{\mathbf{w}}_{e}^{T}(x) \overline{\mathbf{p}}_{e}(x) d x=\delta \overline{\mathbf{q}}_{e}^{T} \overline{\mathbf{P}}_{e}, \quad \int_{0}^{l_{e}} \delta \overline{\mathbf{w}}_{e}^{T}(x) \overline{\mathbf{b}}_{e}(x) d x=-s^{2} \delta \overline{\mathbf{q}}_{e}^{T} \mathbf{M}_{e} \overline{\mathbf{q}}_{e},
$$

where $\overline{\mathbf{P}}_{e}$ is the Laplace transform of nodal excitation forces and $\mathbf{M}_{e}$ is the well-known elemental mass matrix of the rod. Moreover, the right hand size of Eq. (17) could be written, using relationships (12.2) and (14), in the form:

$$
\int_{0}^{l_{e}} \delta \overline{\mathbf{e}}^{T}(x) \overline{\mathbf{s}}(x) d x=\delta \overline{\mathbf{q}}_{e}^{T} \widetilde{\mathbf{K}}_{e} \overline{\mathbf{q}}_{e},
$$

where the elemental viscoelastic stiffness matrix is

$$
\widetilde{\mathbf{K}}_{e}=\int_{0}^{l_{e}} \mathbf{B}^{T}(x) s \overline{\mathbf{G}}(s) \mathbf{B}(x) d x .
$$

It is useful to divide the above viscoelastic stiffness matrix into two matrixes: the elemental elastic stiffness matrix $\mathbf{K}_{e}$ and the second matrix $\mathbf{K}_{e v}$ which will be called the elemental viscous matrix. To be exact, the last matrix expresses the damping properties of the rod only when the classic Zener model (i.e. when $\alpha=1$ in Eq. (4) and (5)) is used in the constitutive equation (14). Taking into account relationships (7), it is possible to write

where

$$
\widetilde{\mathbf{K}}_{e}=\mathbf{K}_{e}+\mathbf{K}_{e v}=(1+\theta(s)) \mathbf{K}_{e},
$$




$$
\mathbf{K}_{e v}=\frac{\tau^{\alpha} s^{\alpha}}{1+\tau^{\alpha}{ }^{\alpha}{ }^{\alpha}} \frac{\Delta E}{E_{0}} \int_{0}^{l_{e}} \mathbf{B}^{T}(x) \mathbf{E}_{0} \mathbf{B}(x) d x, \quad \mathbf{K}_{e}=\int_{0}^{l_{e}} \mathbf{B}^{T}(x) \mathbf{E}_{0} \mathbf{B}(x) d x,
$$

are the above-mentioned elemental viscous matrix and the well-known elemental stiffness matrix of the elastic rod made of an elastic material of which the elastic module is $E_{0}$, respectively. Moreover, $\mathbf{E}_{0}=E_{0} \operatorname{diag}(A, J)$. It is important to note that both the abovementioned matrices are proportional and the factor of proportionality is $\theta(s)$.

Finally, from the virtual work equation (17), the following equation for the finite element

is obtained.

$$
\overline{\mathbf{R}}_{e}=(1+\theta(s)) \mathbf{K}_{e} \overline{\mathbf{q}}_{e}+s^{2} \mathbf{M}_{e} \overline{\mathbf{q}}_{e}-\overline{\mathbf{P}}_{e},
$$

\section{Dynamic properties of viscoelastic frame structures}

Now it is assumed that the whole frame structure is made of a homogenous viscoelastic material and one rheological model is used to describe the mechanical properties of the material. Applying the usual finite element procedure, the equation of motion of structure in the Laplace domain can be written in the following form:

$$
\left\{[1+\theta(s)] \mathbf{K}+s^{2} \mathbf{M}\right\} \overline{\mathbf{q}}=\mathbf{P},
$$

where $\overline{\mathbf{q}}$ and $\overline{\mathbf{P}}$ are global vectors of the Laplace transform of nodal displacements and excitation forces, respectively. Moreover, $\mathbf{M}$ and $\mathbf{K}$ are the global mass matrix and the global stiffness matrix of the elastic frame, respectively. The stiffness matrix $\mathbf{K}$ is built using the elemental matrices given by Eq. (22.2).

The dynamic properties of viscoelastic structure are obtained assuming, firstly, that $\overline{\mathbf{P}}=\mathbf{0}$, and solving the nonlinear eigenvalue problem

$$
\left\{[1+\theta(s)] \mathbf{K}+s^{2} \mathbf{M}\right\} \overline{\mathbf{q}}=\mathbf{0},
$$

where $S$ is the eigenvalue and $\overline{\mathbf{q}}$ is the eigenvector.

In a general case and when the properties of viscoelastic elements of structure are modelled with the help of classic rheological models, the modes of vibration are complex and are also called the viscous or elastic modes of vibration, except that, for some rheological models (for example: the Zener and the generalized Maxwell model), the real mode of vibration, also called the non-viscous mode, exists. However, for the fractional Zener model, the real mode of vibration does not exist [3].

It is difficult to solve the nonlinear eigenvalue problem of this type. Fortunately, in the considered case the eigenvalue problem (25) can easily be transformed into the following linear eigenvalue problem

$$
\left(\mathbf{K}-\omega_{e}^{2} \mathbf{M}\right) \overline{\mathbf{q}}_{l}=\mathbf{0},
$$

where $\overline{\mathbf{q}}_{l}$ is the eigenvector and $\omega_{e}$ the eigenvalue of the linear eigenvalue problem (26) and linked with the eigenvalue of the nonlinear problem by

$$
f(s)=s^{2}+\omega_{e}^{2}(1+\theta(s))=0 .
$$


It is easy to recognize that the linear eigenvalue problem (26) is identical to the eigenvalue problem from which the natural frequency of the considered structure is calculated but is made of an elastic material with the elastic modulus $E_{0}$. Many wellknown procedures [1] can be used to solve the linear eigenvalue problem (26).

The matrices $\mathbf{K}$ and $\mathbf{M}$ in Eqns (25) and (26) are positive definite ones, which means that the eigenvalue $\omega_{e}^{2}$ is a real, positive number and the eigenvector $\overline{\mathbf{q}}_{l}$ is a real vector. Moreover, since $\overline{\mathbf{q}}=\overline{\mathbf{q}}_{l}$, the eigenvector of the nonlinear eigenvalue problem (25) is also a real vector. In addition, two eigenvectors of this kind (say, $\overline{\mathbf{q}}_{i}$ and $\overline{\mathbf{q}}_{j}$ ), fulfil the wellknown orthogonality conditions:

$$
\overline{\mathbf{q}}_{i}^{T} \mathbf{M} \overline{\mathbf{q}}_{j}=\delta_{i j}, \quad \overline{\mathbf{q}}_{i}^{T} \mathbf{K} \overline{\mathbf{q}}_{j}=\omega_{e i}^{2} \delta_{i j},
$$

where $\delta_{i j}$ is the Kronecker's symbol.

However, the eigenvalues of the above mentioned problems (25) and (26) are different because $s$ must be determined from Equation (27). It is a nonlinear algebraic equation and, for a given $\omega_{e}$, it has more than one solution. Numerical experiments show that Eq. (27) has two complex, conjugate solutions in the case $\alpha \neq 1$ and three solutions (two complex, conjugate solutions and one real solution) when $\alpha=1$.

In the case of elastic structures with $n$-th degree of freedom, we have $n$ eigenvalues and eigenvectors but there are more than $n$ solutions to the eigenvalue problem for viscoelastic structures. When the fractional Zener $(\alpha \neq 1)$ is used, it is $2 n$ eigenvalues but in the case of the classic Zener model $(\alpha=1)$ we have $3 n$ eigenvalues. Because the number of eigenvectors $\overline{\mathbf{q}}$ and $\overline{\mathbf{q}}_{l}$ is $n$, we conclude the vector $\overline{\mathbf{q}}$ is in fact a repeated eigenvector corresponding to two or three eigenvalues $s$ determined from Eq. (27) for a given value of $\omega_{e}$.

Having a complex solution $s=\mu+\mathrm{i} \eta$ to Eq. (25), it is possible to calculate the natural frequency $\omega$ and the non-dimensional damping ratio $\gamma$ of the viscoelastic structure using the following formulae:

$$
\omega^{2}=\mu^{2}+\eta^{2}, \quad \gamma=-\mu / \omega
$$

Often the frequency response functions (FRF) are used to characterize the dynamic behaviour of structures. The matrix of FRF is obtained assuming $s=\mathrm{i} \lambda$ and inverting the matrix $\mathbf{D}(\lambda)=[1+\theta(\mathrm{i} \lambda)] \mathbf{K}-\lambda^{2} \mathbf{M}$. For the considered type of structures, this can be done either in a direct way or using a concept similar to that of normal coordinates as described below. For large systems, the second approach is more efficient.

The solution to Eq. (24) for $s=\mathrm{i} \lambda$ is assumed in the form:

$$
\overline{\mathbf{q}}(\mathrm{i} \lambda)=\sum_{j=1}^{n} \mathbf{a}_{j} \bar{x}_{j}(\lambda),
$$

where $\mathbf{a}_{j}$ is the eigenvector fulfilling Eq. (26) and $\bar{x}_{j}(\lambda)$ is the Fourier transform of normal coordinate $x_{j}(t)$ of the elastic structure. After introducing relationship (30) into Eq. (24) multiplied by $\mathbf{a}_{i}^{T}$ and taking into account the orthogonality conditions (28), the following equation is obtained:

from which

$$
\left\{[1+\theta(\mathrm{i} \lambda)] \omega_{e i}^{2}-\lambda^{2}\right\} \bar{x}_{i}=\mathbf{a}_{i}^{T} \mathbf{P}
$$




$$
\bar{x}_{i}=\frac{\mathbf{a}_{i}^{T} \mathbf{P}}{[1+\theta(\mathrm{i} \lambda)] \omega_{e i}^{2}-\lambda^{2}} .
$$

The matrix of FRF $\mathbf{Z}(\mathrm{i} \lambda)=\left\{[1+\theta(\mathrm{i} \lambda)] \mathbf{K}-\lambda^{2} \mathbf{M}\right\}^{-1}$ can now be rewritten in the form:

$$
\mathbf{Z}(\mathrm{i} \lambda)=\sum_{j=1}^{n} \frac{\mathbf{a}_{j} \mathbf{a}_{j}^{T}}{[1+\theta(\mathrm{i} \lambda)] \omega_{e j}^{2}-\lambda^{2}} .
$$

\section{Results of a representative calculation}

The two span beam, shown in Fig. 2, is considered an example. The following data are adopted: the span of the beam $L=4.0 \mathrm{~m}$, the dimension of the beam's cross-section $b=0.2 \mathrm{~m}, h=0.4 \mathrm{~m}$, the unit mass $m=80.0 \mathrm{~kg} / \mathrm{m}$, the parameters of the Zener model are $E_{0}=7.0 \mathrm{MPa}, \tau=0.02 \mathrm{~s}$ and $\alpha=0.7$. The calculation is performed for different ratios $E_{\infty} / E_{0}$ taken in a wide range of $1.1 \div 10.0$. The elastic beam corresponding to the VE one has the modulus $E=7.0 \mathrm{MPa}$.

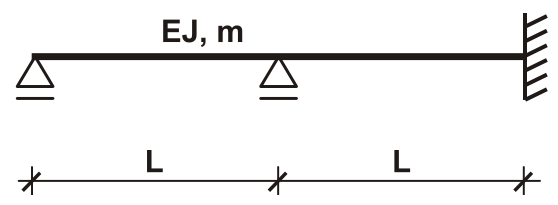

Fig. 2 Diagram of the two-span beam.

The first two natural frequencies of the elastic beam are: $\omega_{1}=6.85 \mathrm{rad} / \mathrm{s}$ and $\omega_{2}=11.87 \mathrm{rad} / \mathrm{s}$. The Newton method is used to solve the nonlinear equation (27). Calculations are performed for different values of $E_{\infty}$ and the results of the calculations are presented in Figs 3 and 4. In Fig. 3, the non-dimensional damping ratio of the first mode (solid line) and of the second mode (dashed line) is presented as a function of the modulus ratio $E_{\infty} / E_{0}$. Both ratios grow first with the $E_{\infty} / E_{0}$ ratio but the non-dimensional damping ratio is approximately constant for ratios higher than 12.0 (for the first mode) and 10.0 (for the second mode).

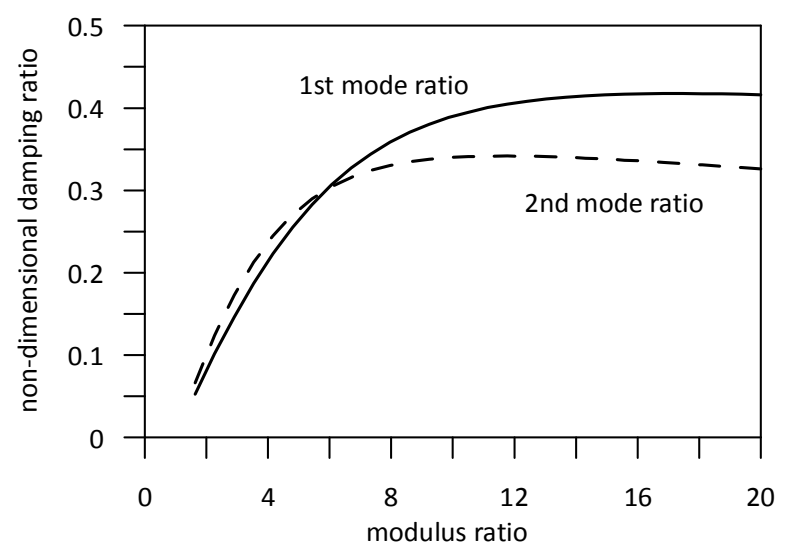

Fig. 3 The non-dimensional damping ratios versus the modulus ratio $E_{\infty} / E_{0}$. 
In Fig. 4, changes of the first and second natural frequencies versus modulus ratio $E_{\infty} / E_{0}$ are shown. Both natural frequencies grow significantly with increasing modulus ratios.

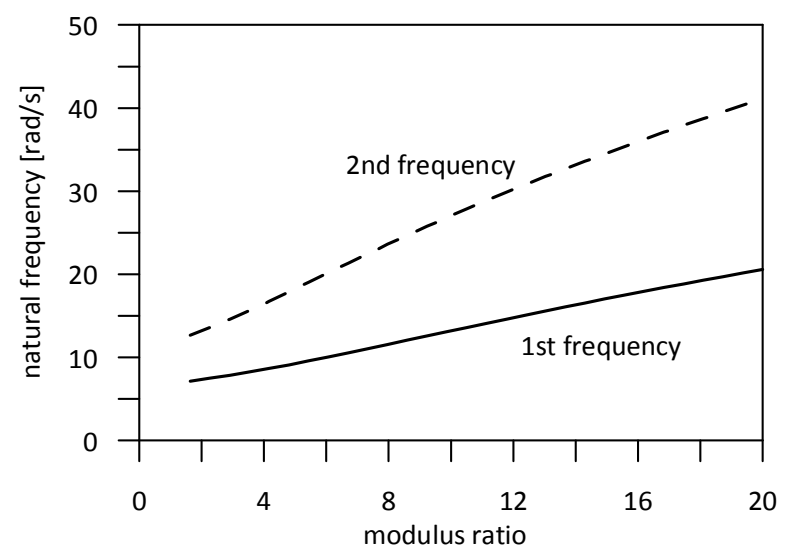

Fig. 4 The natural frequencies versus the modulus ratio $E_{\infty} / E_{0}$.

Finally, it must be stressed that the VE material of the considered beam has very good damping properties.

\section{Concluding remarks}

A method for determining the dynamic characteristics of homogenous VE frame structures, which is computationally very efficient, is presented. The method requires a solution to the linear eigenvalue problem for corresponding elastic structures and a solution to one nonlinear algebraic equation. This makes the method very attractive from the computational point of view. The method is not general but can be applied to a technically important class of structures. It is obvious that the method could also be applied also to other rheological models of VE materials.

This study has been supported by the Poznan University of Technology, the Institute of Structural Engineering, carried out in the year 2019. This support is gratefully acknowledged.

\section{References}

1. K.J. Bathe, Finite Element Procedures in Engineering Analysis, (Prentice-Hall, 1982)

2. R. Lewandowski, Dynamika konstrukcji budowlanych, 2 Ed., (Wydawnictwo Politechniki Poznańskiej, 2018)

3. R. Lewandowski, M. Baum, Arch. Appl. Mech., 85, 1793-1814, (2015)

4. Z. Pawlak, R. Lewandowski, Comput. Struct. 125, 53-61, (2013)

5. S. Adhikari, B. Pascual, J. Vib. Acoust., 132, 021002.1-021002.7. (2011)

6. R. Lewandowski, A. Bartkowiak, H. Maciejewski, Struct. Eng. Mech., 41, 113-137 (2012)

7. K.V. Singh, Int. J. Mech. Sci., 110, 127-137, (2016)

8. R. Lewandowski, P. Wielentejczyk, J. Sound Vibr., 399, 228-243 (2017)

9. F. Mainardi and G. Spada, Creep, relaxation and viscosity properties for basic fractional models in rheology, Eur. Phys. J. Special Topics 193, 133-160 (2011) 